\title{
An efficient calcium phosphate nanoparticle-based nonviral vector for gene delivery
}

This article was published in the following Dove Press journal:

International Journal of Nanomedicine

5 April 201 I

Number of times this article has been viewed

\section{Yachun Liu',2,* \\ Tao Wangl,* \\ Fangli $\mathrm{He}^{1, *}$ \\ Qian Liu',* \\ Dexi Zhang² \\ Shuanglin Xiang' \\ Shengpei $\mathrm{Su}^{2}$ \\ Jian Zhang'}

'Key Laboratory of Protein Chemistry and Developmental Biology, Ministry of Education of China, College of Life Sciences; ${ }^{2}$ Key Laboratory of Chemical Biology and Traditional Chinese Medicine Research and Key Laboratory of Sustainable Resources Processing and Advanced Materials of Hunan Province, Ministry of Education of China, College of Chemistry and Chemical Engineering, Hunan Normal University, Changsha, Hunan, China

*These authors contributed equally to this work
Correspondence: Shuanglin Xiang, Jian Zhang

Key Laboratory of Protein Chemistry and Developmental Biology, Ministry of Education of China, College of Life Sciences, Hunan Normal University, Changsha, Hunan 41008I, China

Tel +86 73I 88872095

Fax +86 73। 88872792

Emailxshlin@hunnu.edu.cn, zhangjian@hunnu.edu.cn
Background: Smaller nanoparticles facilitate the delivery of DNA into cells through endocytosis and improve transfection efficiency. The aim of this study was to determine whether protamine sulfate-coated calcium phosphate (PS-CaP) could stabilize particle size and enhance transfection efficiency.

Methods: pEGFP-C1 green fluorescence protein was employed as an indicator of transfection efficiency. Atomic force microscopy was used to evaluate the morphology and the size of the particles, and an MTT assay was introduced to detect cell viability and inhibition. The classical calcium phosphate method was used as the control.

Results: Atomic force microscopy images showed that the PS-CaP were much smaller than classical calcium phosphate particles. In 293 FT, HEK 293, and NIH 3 T3 cells, the transfection efficiency of PS-CaP was higher than for the classical calcium phosphate particles. The difference in efficiencies implies that the smaller nanoparticles may promote the delivery of DNA into cells through endocytosis and could improve transfection efficiency. In addition, PS-CaP could be used to transfect HEK 293 cells after one week of storage at $4^{\circ} \mathrm{C}$ with a lesser extent of efficiency loss compared with classical calcium phosphate, indicating that protamine sulfate may increase the stability of calcium phosphate nanoparticles. The cell viability inhibition assay indicated that both nanoparticles show similar low cell toxicity.

Conclusion: PS-CaP can be used as a better nonviral transfection vector compared with classical calcium phosphate.

Keywords: transfection efficiency, particle size, protamine sulfate, stability

\section{Introduction}

Calcium phosphate is one of the widely used nonviral vectors for in vitro transfection of a variety of mammalian cells due to its low toxicity, biodegradability, ease of use, and adsorptive capacity for pDNA. ${ }^{1-4}$ However, the transfection efficiency of the classical calcium phosphate method strongly depends on preparation parameters, such as $\mathrm{pH}$, concentrations of calcium chloride and DNA, temperature, the time between precipitation and transfection, cell types, as well as the skills of the researcher. ${ }^{4-6}$ Thus, the reproducibility of this method is poor compared with other transfection methods based on liposomes and polymers, and has to be optimized for every cell line and laboratory. ${ }^{1,7}$ Furthermore, calcium phosphate particles grow with time, and consequently, its transfection efficiency is reduced. ${ }^{1}$

The size of calcium phosphate particles greatly affects their transfection efficiency. ${ }^{8}$ Many efforts have been made to prepare calcium phosphate particles with appropriate sizes, including the preparation of calcium phosphate nanoparticles to facilitate 
delivery of DNA into cells through endocytosis and thereby improve transfection reproducibility and efficiency. ${ }^{9}$ Olton et al reported a novel method to improve the manufacture of the calcium phosphate precipitate by optimizing both the stoichiometry $(\mathrm{Ca} / \mathrm{P}$ ratio $)$ of the calcium phosphate particles, as well as the mode by which the calcium and phosphate precursor solutions are mixed..$^{10}$ Additionally, maximum transfection efficiencies in both HeLa and MC3T3-E1 cell lines have been obtained when the synthesized particles used were within the optimum $\mathrm{Ca} / \mathrm{P}$ ratio between 100 and 300 , and with sizes between 25 and $50 \mathrm{~nm}$. Bisht et al and Roy et al condensed DNA in a reverse microemulsion environment to prepare calcium phosphate nanoparticles $100-120 \mathrm{~nm}$ in diameter. ${ }^{11,12}$ The in vitro transfection efficiency of these calcium phosphate nanoparticles was found to be higher than that of the commercial transfection reagent, Polyfect ${ }^{\circledR}$. However, these particles aggregated rapidly with time.

Functionalization by organic molecules was therefore used to preserve the small size of the calcium phosphate particles and to inhibit their further growth. A novel calcium phosphate nanoparticle formulation was developed by rapid precipitation, followed by immediate adsorption of DNA, which also stabilizes calcium phosphate colloids. The DNA-coated calcium phosphate nanoparticles were 10-20 $\mathrm{nm}$ in diameter, showed good transfection efficiency, and could be stored for weeks without loss of their transfection activity. ${ }^{7,8,13,14}$ Moreover, the efficiency of the multishell particles is significantly higher than that of simple DNA-coated calcium phosphate nanoparticles. ${ }^{1}$ A lipid-coated calcium phosphate nanoparticle formulation was developed for systemic siRNA and gene delivery. ${ }^{9}$ Kakizawa et al prepared nanoparticles consisting of calcium phosphate, DNA, and block copolymers. Small particle sizes and good colloidal stability were achieved by the steric effect of a polyethylene glycol layer surrounding the calcium phosphate core. ${ }^{15,16}$ Liu et al reported the in vitro preparation of 24-35 nm calcium phosphate nanoparticles coated with bovine serum albumin as a DNA vector. ${ }^{17}$ Chowdhury et al successfully constructed nanohybrids of DNA/calcium phosphate particles with extracellular matrix proteins (either collagen 1 or fibronectin), leading to remarkably high transgenic expression in mammalian cells. ${ }^{18}$

Cationic cell-penetrating peptides have been shown to deliver a wide variety of cargo into primary cells and most tissues in preclinical models. ${ }^{19}$ Protamine sulfate is a $5 \mathrm{kDa}$ cationic peptide rich in arginine that is similar to cationic cell-penetrating peptides. ${ }^{20}$ In the present study, considering that the adsorption of protamine sulfate onto the surface of the DNA/calcium phosphate particles may stabilize particle size and further inhibit growth, we have developed a novel protamine sulfate-modified calcium phosphate nanoparticle formulation (PS-CaP) for efficient delivery of DNA into cells.

\section{Materials and methods Materials}

All chemicals and cell culture media used in these experiments were purchased as follows: analytical protamine sulfate (99.0\%) from Sigma Aldrich (St Louis, MO), other compounds of analytical grade from Shanghai Songon, China, and Dulbecco's minimum essential medium (DMEM) and penicillin/streptomycin from Invitrogen. The three cell lines used in these experiments, namely, 293 FT, HEK 293, and NIH 3 T3 cells, were obtained from the American Type Culture Collection (Rockville, MD).

\section{Synthesis of pDNA-loaded classical calcium phosphate and PS-CaP}

The pDNA-loaded classical calcium phosphate was synthesized according to the methods reported in the literature, ${ }^{3,4}$ as follows: protamine sulfate was dissolved in steaming water up to concentrations of $1 \%, 2 \%$, and $4 \%$, and then $\mathrm{pH}$ was adjusted to 7.0. Solution 1 was obtained by $2 \times$ HEPES-buffered saline and protamine sulfate solution mixed at a volume ratio of 1:1. pEGFP-C1 green fluorescence protein was incubated with $0.25 \mathrm{M}$ of calcium chloride for five minutes and was then added to solution 1 on a vortex at a rate of about $1 \mathrm{drop} / \mathrm{sec}$. The classical calcium phosphate particles and PS-CaP particles were designated as 0PS-CaP, 1PS-CaP, 2PS-CaP, and 4PS-CaP with $0 \%, 1 \%, 2 \%$, and $4 \%$ protamine sulfate concentrations, respectively. After different periods of storage at $4^{\circ} \mathrm{C}$, the complexes were used for transfection.

\section{Atomic force microscopy}

A Nanoscope IIIa controller with a multimode atomic force microscope (Digital Instruments, Santa Barbara, CA) was used for imaging. Atomic force microscopic images were taken in air under tapping mode with standard $125 \mathrm{~mm}$ single-crystal silicon cantilevers (Model TESP, Digital Instruments). All images shown here are height images.

\section{In vitro transfection studies using $293 \mathrm{FT}$, HEK 293, and NIH 3T3 cells}

The 293 FT, HEK 293, and NIH 3T3 cell lines were cultured in $10 \%$ fetal calf serum/DMEM supplemented with 
$100 \mathrm{IU} / \mathrm{mL}$ penicillin and $100 \mu \mathrm{g} / \mathrm{mL}$ streptomycin under a humidified atmosphere at $37^{\circ} \mathrm{C}$ and $5 \% \mathrm{CO}_{2}$. The cells were maintained at subconfluence and passaged every 2-3 days. The cells were then seeded into 24 -well cell culture plates at a cell concentration of $1.63 \times 10^{5}$ cell/well a day before transfection so that a cell confluence of approximately $70 \%$ could be obtained just prior to transfection. The resulting transfection mixtures were then immediately added to the wells $(50 \mu \mathrm{L} /$ well) in a dropwise manner. The PS-CaP particles and classical calcium phosphate particles were allowed to remain in the cell culture medium for 4-6 hours, after which the cell culture medium was replaced with fresh serum medium, and the cells were allowed to incubate for another 24 hours. At 24 hours post-transfection, the transfection result of the reporter gene selected, $p E G F P-C 1$, was observed under confocal fluorescence microscopy (Radiance 2100, Zeiss).

\section{MTT assay}

HEK 293 cells were harvested during the logarithmic growth phase and then seeded into 24-well plates (Corning Glassworks, Corning, NY) at a density of $1.5 \times 10^{4}$ cells $/ \mathrm{mL}$ in a final volume of $500 \mu \mathrm{L} /$ well. The cells were grown to $90 \%$ confluence and were then treated with the PS-CaP and classical calcium phosphate particles using a series of dosages in low serum for a further 24 hours. Then, $50 \mu \mathrm{L}$ of 3-(4.5dimethylthiazol-2-yl)-2,5-diphenyltetrazolium bromide (MTT, Sigma Aldrich) solution $(5 \mathrm{mg} / \mathrm{mL}$ in phosphatebuffered saline) was added to each well for an additional four hours. Dark blue formazan crystals were formed in the intact cells. The supernatant was removed, and the crystals were solubilized with $100 \mu \mathrm{L}$ dimethylsulfoxide (Sigma Aldrich). Cell viability was determined by a conventional MTT reduction assay. This assay is based on the reduction of MTT into formazan, which provides a sensitive measurement of the normal metabolic status of cells. The extent of MTT reduction was measured using a Model 680 microplate reader (Bio-Rad, Bath, UK) at $570 \mathrm{~nm}$. The experiments were performed at least three times.

\section{Flow cytometry analysis}

The fluorescence intensity of the pEGFP-C1 green fluorescence protein after transgenic expression was measured by flow cytometry. The cells were gently harvested using $0.2 \%$ trypsin-ethylenediamine tetra-acetic acid solution at 24 hours post-transfection, washed twice with $3 \mathrm{~mL}$ phosphate-buffered saline, and $1 \times 10^{4}$ events were analyzed on a flow cytometer equipped with a $488 \mathrm{~nm}$ excitation laser wavelength operated at $450 \mathrm{~V}$. The untransfected cells were gated as a population in M1 on a flow cytometry histogram, and the transfected cells were gated as a population in $M 2$. The transfection efficiencies of the pEGFP-C1 green fluorescence protein were estimated at the percentage of $\mathrm{M} 2 /(\mathrm{M} 1+\mathrm{M} 2)$.

\section{Statistical analysis}

Statistically significant differences between the control and treatment groups were determined by the Student's $t$-test. The accepted level of significance was $P<0.05$. All experiments were conducted in triplicate with separate independent cultures.

\section{Results}

\section{Size and morphology of calcium phosphate particles}

The size and morphology of the calcium phosphate particles were measured using atomic force microscopy. Figure 1 shows the atomic force microscopic images for the 0PS-CaP and the 1PS-CaP after storage for two hours and seven days at $4^{\circ} \mathrm{C}$, respectively. Nanoparticles with nearly spherical morphology were observed. The atomic force microscopic images also revealed that the 1PS-CaP were much smaller than the 0PS-CaP after different storage periods at $4{ }^{\circ} \mathrm{C}$. The primary sizes of the $1 \mathrm{PS}-\mathrm{CaP}$ and the OPS-CaP were approximately $30 \mathrm{~nm}$ and $150 \mathrm{~nm}$ after storage for two hours, respectively. After storage for seven days, the sizes of both grew to approximately $100 \mathrm{~nm}$ and $500 \mathrm{~nm}$, respectively.

\section{In vitro transfection studies in mammalian cell lines}

In vitro transfection of the NIH 3T3, 293 FT, and HEK 293 cells with the pEGFP-C1 green fluorescence protein encapsulated in the 1PS-CaP, 2PS-CaP, 4PS-CaP, and OPS-CaP was observed using laser fluorescence confocal microscopy. pEGFP-C1 green fluorescence protein was used as an indicator of transfection efficiency, and the classical calcium phosphate method was used as the control. As shown in Figures 2 and 3, as well as in the Table 1, the protamine sulfate-modified particles show significantly higher transfection efficiency than the 0PS-CaP for the three cell lines. Furthermore, their transfection efficiencies were enhanced with increasing protamine sulfate concentrations.

To determine further the effects of protamine modification on the in vitro transfection of mammalian cells, the 1PS-CaP, 

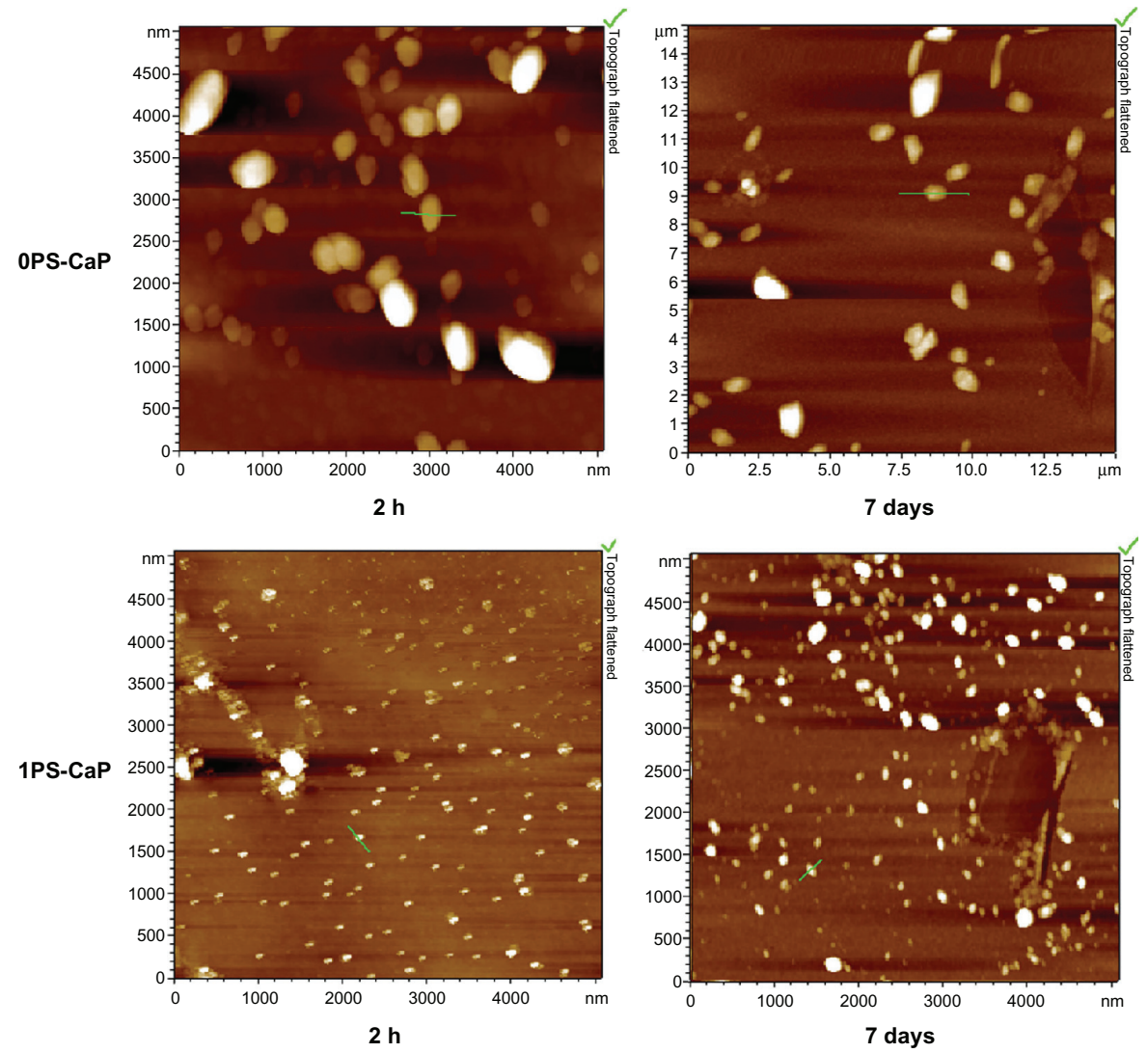

Figure I Representative atomic force microscopic images of the classical calcium phosphate particle (OPS-CaP) and protamine sulfate-coated calcium phosphate particle (IPS-CaP) after standing for two hours and seven days, respectively.

Abbreviations: PS-CaP, protamine sulfate coated-calcium phosphate; OPS-CaP, PS-CAP with 0\% protamine sulfate concentration; IPS-CaP, PS-CAP with I\% protamine sulfate concentration.

2PS-CaP, 4PS-CaP, and 0PS-CaP were used for transfection of HEK 293 cells after they were left to stand for two hours and for seven days. As shown in Figure 3 and in the Table, there was some decrease in their transfection efficiencies. After standing for seven days, the pEGFP-C1 green fluorescence of the 0PS-CaP for transfection of HEK 293 significantly declined, whereas the protamine sulfate-modified particles remained with higher transfection efficiencies.

\section{Cytotoxicity assay}

Given that this present work aimed to use the PS-CaP particles as gene delivery carriers, their cytotoxicity was considered. Figure 4 shows the effect of the PS-CaP and 0PS$\mathrm{CaP}$ dosage on cell viability, as detected by MTT assay. Cell viability decreased with increasing dosage in HEK 293 cells. However, their MTT values did not decline significantly and were not distinctly different. In addition, the results of the statistical analyses show that the difference between the control (0PS-CaP) and treatment groups (PS-CaP) was not significant at the 0.05 level except for $1 \mathrm{PS}-\mathrm{CaP}$ and $0 \mathrm{PS}-\mathrm{CaP}$ at the $50 \mu \mathrm{L}$ dosage $(P<0.05)$. These results indicate that
PS-CaP have low cytotoxicity like 0PS-CaP when near the transfection dosage.

\section{Discussion}

The search for efficient and safe carriers to deliver genes into cells remains a great challenge in gene therapy. Inorganic nanoparticles are especially suitable for this purpose because they can be surface-functionalized in many different ways, and thus show good biocompatibility and potential for targeted delivery. Furthermore, nanoparticles can penetrate the cell wall via endocytosis and deliver genes or biomolecules into living systems, usually for therapeutic purposes. Many inorganic nanoparticles, such as calcium phosphate, gold, carbon materials, silicon oxide, iron oxide, and layered double hydroxide, have been studied for gene delivery. ${ }^{21,22}$ Among these nonviral gene delivery vectors, the coprecipitation of calcium phosphate with pDNA remains an attractive option due to the good biocompatibility and biodegradability of calcium phosphate. ${ }^{10}$

The particle size of calcium phosphate significantly affects transfection efficiency. ${ }^{8}$ However, calcium phosphate 


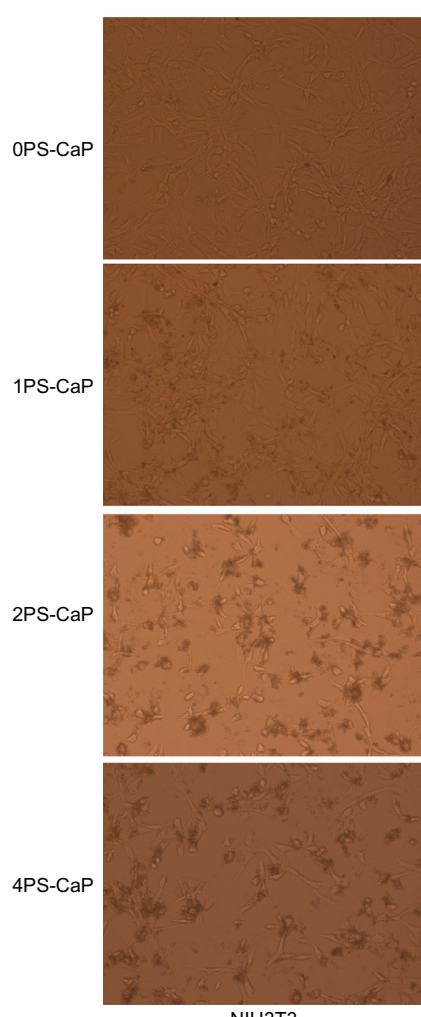

$\mathrm{NIH} 3 \mathrm{T3}$
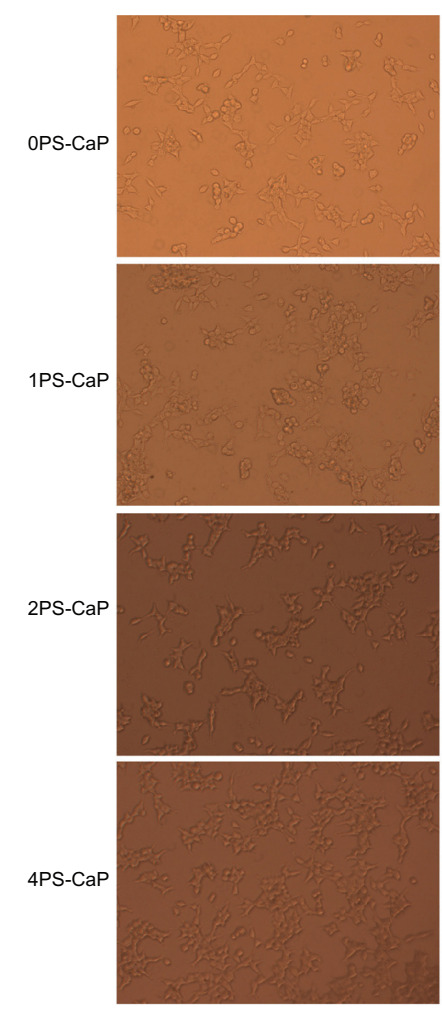

$293 \mathrm{FT}$

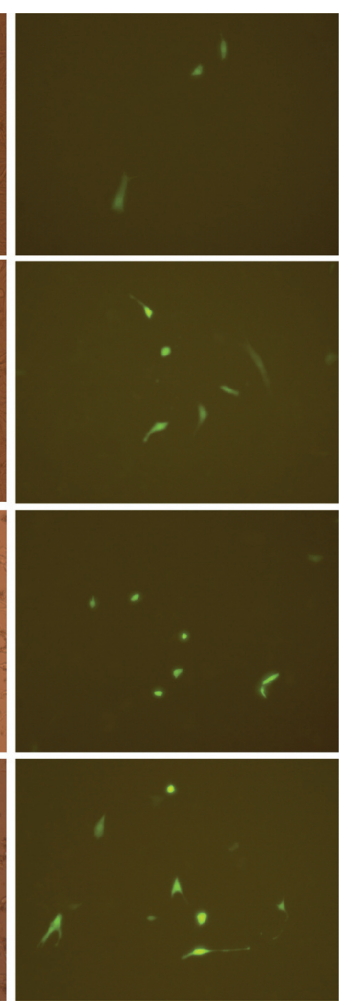

$24 \mathrm{~h}$
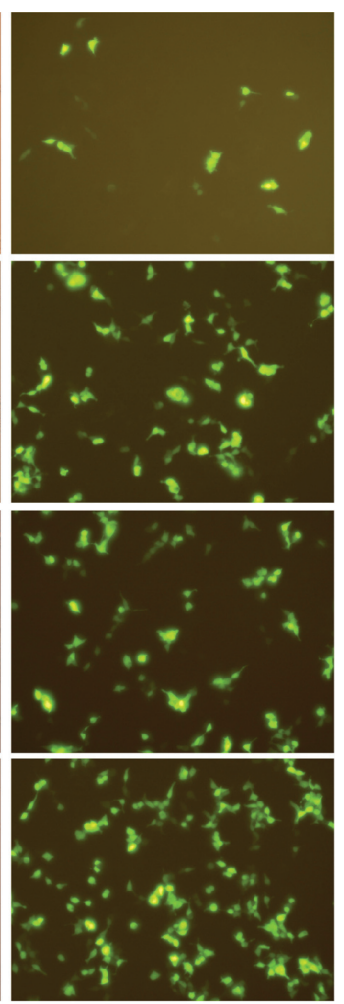

$24 \mathrm{~h}$
Figure 2 Fluorescence confocal microscopy (20× magnification) of NIH 3T3 and 293 FT cells transfected with the classical calcium phosphate particle (OPS-CaP) and IPS-CaP, 2PS-CaP, and 4PS-CaP.

Abbreviations: PS-CaP, protamine sulfate coated-calcium phosphate; OPS-CaP, PS-CAP with $0 \%$ protamine sulfate concentration; IPS-CaP, PS-CAP with $1 \%$ protamine sulfate concentration; $2 \mathrm{PS}-\mathrm{Cap}, \mathrm{PS}-\mathrm{CaP}$ with $2 \%$ protamine sulfate concentration; 4PS-CaP, PS-CAP with $4 \%$ protamine sulfate concentration.

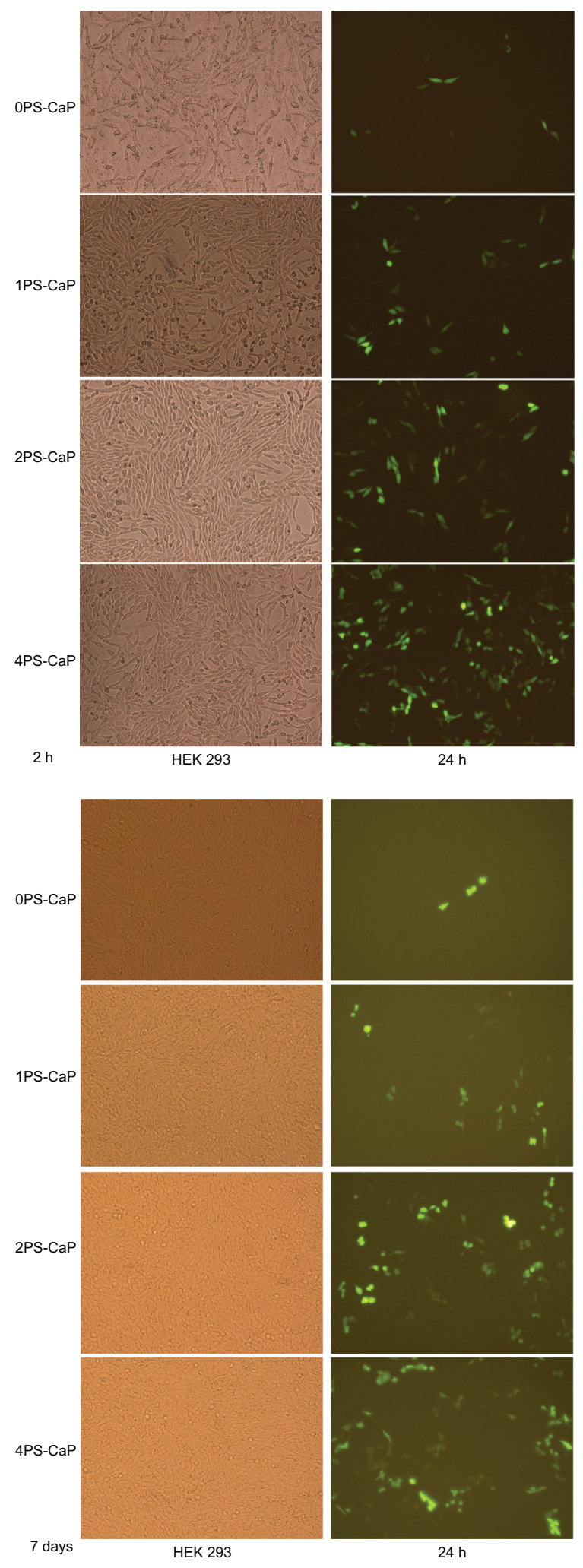

Figure 3 Fluorescence confocal microscopy (magnification 20x in all cases) of HEK 293 cells that were transfected with the classical calcium phosphate particle (OPS$\mathrm{CaP}$ ) and IPS-CaP, 2PS-CaP, and 4PS-CaP, after standing for two hours and for seven days.

Abbreviations: PS-CaP, protamine sulfate-coated calcium phosphate; OPS-CaP, PS-CAP with $0 \%$ protamine sulfate concentration; IPS-CaP, PS-CAP with $1 \%$ protamine sulfate concentration; $2 \mathrm{PS}-\mathrm{Cap}, \mathrm{PS}-\mathrm{CaP}$ with $2 \%$ protamine sulfate concentration; 4PS-CaP, PS-CAP with $4 \%$ protamine sulfate concentration. 
Table I Results of all transfection experiments

\begin{tabular}{llllll}
\hline Cell type & Storage time & \multicolumn{3}{l}{ Transfection efficiency (\%)* } & 2PS-CaP \\
\cline { 3 - 6 } & & OPS-CaP & IPS-CaP & 6.15 & 4PS-CaP \\
\hline HEK 293 & 2 hours & 2.64 & 3.57 & 3.64 & 8.33 \\
& 7 days & 1.08 & 1.94 & 22.81 & 4.94 \\
293 FT & 20 minutes & 3.98 & 15.56 & 3.81 & 49.43 \\
NIH 3T3 & 20 minutes & 0.75 & 1.97 & 4.71 \\
\hline
\end{tabular}

Note: *Transfection efficiency was computed by calibration of blank value from the result of flow cytometry analysis.

particles grow with time, and, accordingly, their transfection efficiency decreases. ${ }^{1}$ Many efforts have been made to prepare stabilized calcium phosphate nanoparticles to improve their transfection reproducibility and efficiency by surface-functionalized modification. ${ }^{9,21,22}$ For example, remarkably high transgenic expression in mammalian cells has been achieved by the transfection of DNA/calcium phosphate particle nanohybrids modified with extracellular matrix proteins (either collagen 1 or fibronectin). ${ }^{18}$

Cationic cell-penetrating peptides have been shown to deliver a wide variety of cargo into primary cells and most tissues in preclinical models. ${ }^{19}$ Protamine sulfate is a $5 \mathrm{kDa}$ cationic peptide rich in arginine residues that is similar to Capps.$^{20}$ In the present study, considering that the protamine sulfate-coated surface of the DNA/calcium phosphate particles may stabilize their size and inhibit their further growth, we developed a PS-CaP nanoparticle formulation for efficient delivery of DNA into cells.

Surface charges and the steric effect of surface functionalization of the shell layer that circumvents the inorganic nanoparticle core play important roles in regulating particle size and colloidal stability, and therefore affect

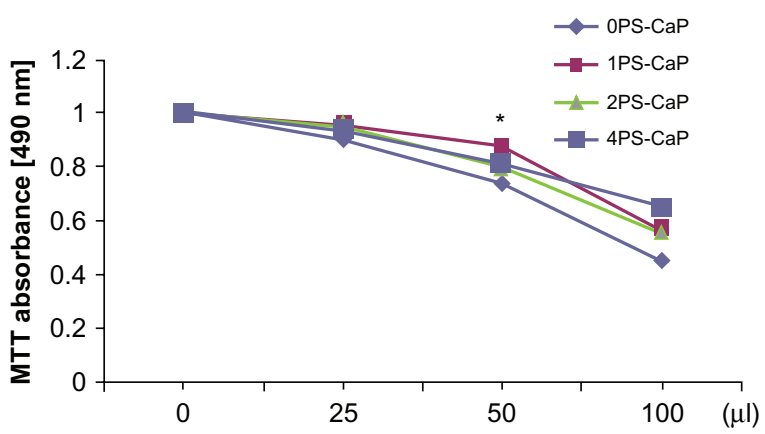

Figure 4 Effect of PS-CaP and classical calcium phosphate particle (OPS-CaP) dosage on cell viability. A series of different doses ( $0-2$ times that of transfection dosage) of the OPS-CaP and IPS-CaP, 2PS-CaP, and 4PS-CaP were used to treat HEK 293 cells, and their inhibitory effect on cell proliferation was evaluated by measuring MTT absorption.

Note: *Indicates that IPS-CaP is significantly different from OPS-CaP $(P<0.05)$ Abbreviations: PS-CaP, protamine sulfate-coated calcium phosphate; OPS-CaP, PSCAP with $0 \%$ protamine sulfate concentration; IPS-CaP, PS-CAP with $1 \%$ protamine sulfate concentration; $2 \mathrm{PS}-\mathrm{Cap}, \mathrm{PS}-\mathrm{CaP}$ with $2 \%$ protamine sulfate concentration; $4 \mathrm{PS}-\mathrm{CaP}, \mathrm{PS}-\mathrm{CAP}$ with $4 \%$ protamine sulfate concentration. transfection efficiency. ${ }^{7,21}$ In the present study, the steric effect of the adsorption of protamine sulfate onto the surface of the DNA/calcium phosphate particles may play an important role in stabilizing their primary sizes and inhibiting their further growth. This effect is due to the possible existence of an optimum zeta potential for the adhesive interactions with cell membranes, which drives the nanoparticles to approach the cell surface and promote colloidal particle stability by electrostatic interaction. ${ }^{21,23}$ This zeta potential is generally close to $20-30 \mathrm{mV}$. However, the zeta potential of the PS-CaP used in the study is approximately $13.2 \mathrm{mV}$, ie, slightly higher than that of the 0PS-CaP (about $9.5 \mathrm{mV}$ ). Moreover, both values are lower than the optimum zeta potential. Thus, the primary size of the PS-CaP was much smaller than that of the 0PS-CaP, possibly because of the steric effect derived from the protamine sulfate-coated surface of the DNA/calcium phosphate particles. This steric effect also simultaneously inhibits their further growth, thereby enhancing their storage stability.

Smaller nanoparticles favor delivery of DNA to cells through endocytosis and accordingly improve transfection reproducibility and efficiency. ${ }^{9}$ Atomic force microscopic imaging shows that the PS-CaP were much smaller than the OPS-CaP particle (Figure 1). As shown in Figures 2 and 3, as well as in the Table, the transfection efficiency of PS-CaP was clearly higher than with the classical calcium phosphate method in 293 FT, HEK 293, and NIH 3 T3 cells. This difference implies that smaller nanoparticles may promote delivery of DNA into cells through endocytosis, and could improve the transfection efficiency. In addition, Figure 3 shows that PS-CaP could be used for transfection after one week of storage at $4^{\circ} \mathrm{C}$ with less efficiency loss, indicating that protamine sulfate may increase the storage stability of calcium phosphate nanoparticles.

Calcium phosphate is the most important inorganic constituent of biological hard tissues in living organisms. ${ }^{21}$ Calcium phosphate coprecipitated with DNA has become one of the most commonly used nonviral vectors since it was initially introduced by Graham and van der Eb. ${ }^{2}$ Its excellent biocompatibility is very important for gene delivery, and 
therefore its cytotoxicity has to be evaluated. The cell viability inhibition assay indicated that PS-CaP have low cell toxicity, similar to 0PS-CaP (Figure 4). In conclusion, PS-CaP are better nonviral transfection vectors than the $0 \mathrm{PS}-\mathrm{CaP}$ vector.

\section{Conclusion}

We have developed a novel PS-CaP nanoparticle formulation for the delivery of DNA into cells. Protamine sulfate was shown to stabilize the size of DNA/calcium phosphate particles, inhibit their further growth, and promote endocytic delivery of DNA into cells, thereby improving transfection efficiency. Furthermore, PS-CaP have low cell toxicity, similar to OPS-CaP. Therefore, PS-CaP may be used as a better nonviral transfection vector compared with the OPS-CaP vector.

\section{Acknowledgments}

This study was supported by the 863 Project of the Ministry of Science and Technique of China (Grant 2007AA02Z172), the Postdoctoral Science Foundation of China (Grant 20100471217), the Scientific Research Fund of Hunan Provincial Education Department (Grant 09C621), the Postdoctoral Science Research Fund of Hunan Provincial Science and Technique Department (Grant 2009RS3015), the Open Fund of the Key Laboratory of the Chemical Biology and Traditional Chinese Medicine Research (Ministry of Education of China), and Hunan Normal University (Grant KLCBTCMR2009-12).

\section{Disclosure}

The authors report no conflicts of interest in this work.

\section{References}

1. Sokolova V, Radtke I, Heumann R, Epple M. Effective transfection of cells with multi-shell calcium phosphate-DNA nanoparticles. Biomaterials. 2006;27:3147-3153.

2. Graham FL, van der Eb AJ. A new technique for the assay of infectivity of human adenovirus 5 DNA. Virology. 1973;52:456-467.

3. Jordan M, Schallhorn A, Wurm FM. Transfecting mammalian cells: Optimization of critical parameters affecting calcium-phosphate precipitate formation. Nucleic Acids Res. 1996;24:596-601.

4. Jordan M, Wurm FM. Transfection of adherent and suspended cells by calcium phosphate. Methods. 2004;33:136-143.

5. Seelos C. A critical parameter determining the aging of DNA-calcium phosphate precipitates. Anal Biochem. 1997;245:109-111.
6. Urabe M, Kume A, Tobita K, Ozawa K. DNA/calcium phosphate precipitates mixed with medium are stable and maintain high transfection efficiency. Anal Biochem. 2000;278:91-92.

7. Pedraza C, Bassett D, McKee M, Nelea V, Gbureck U, Barralet J. The importance of particle size and DNA condensation salt for calcium phosphate nanoparticle transfection. Biomaterials. 2008;29: 3384-3392.

8. Welzel T, Radtke I, Meyer-Zaika W, Heumann R, Epple M. Transfection of cells with custom-made calcium phosphate nanoparticles coated with DNA. J Mater Chem. 2004;14:2213-2217.

9. Li J, Chen Y, Tseng Y, Mozumdar S, Huang L. Biodegradable calcium phosphate nanoparticle with lipid coating for systemic siRNA delivery. J Control Release. 2010;142:416-421.

10. Olton D, Li J, Wilson ME, et al. Nanostructured calcium phosphates (NanoCaPs) for non-viral gene delivery: Influence of the synthesis parameters on transfection efficiency. Biomaterials. 2007;28: 1267-1279.

11. Roy I, Mitra S, Maitra A, Mozumdar S. Calcium phosphate nanoparticles as novel non-viral vectors for targeted gene delivery. Int $J$ Pharm. 2003;250:25-33.

12. Bisht S, Bhakta G, Mitra S, Maitra A. pDNA loaded calcium phosphate nanoparticles: Highly efficient non-viral vector for gene delivery. Int $J$ Pharm. 2005;288:157-168.

13. Eufinger H, Rasche C, Lehmbrock J, et al. Performance of functionally graded implants of polylactides and calcium phosphate/calcium carbonate in an ovine model for computer assisted craniectomy and cranioplasty. Biomaterials. 2007;28:475-485.

14. Welzel T, Meyer-Zaika W, Epple M. Continuous preparation of functionalised calcium phosphate nanoparticles with adjustable crystallinity. Chem Commun. 2004;10:1204-1205.

15. Kakizawa Y, Kataoka K. Block copolymer self-assembly into monodispersive nanoparticles with hybrid core of antisense DNA and calcium phosphate. Langmuir. 2002;18:4539-4543.

16. Kakizawa Y, Miyata K, Furukawa S, Kataoka K. Size-controlled formation of a calcium phosphate-based organic-inorganic hybrid vector for gene delivery using poly(ethylene glycol)-block-poly(aspartic acid). Adv Mater. 2004;16:699-702.

17. Liu T, Tang A, Zhang G, et al. Calcium phosphate nanoparticles as a novel nonviral vector for efficient transfection of DNA in cancer gene therapy. Cancer Biother Radiopharm. 2005;20:141-149.

18. Chowdhury EH, Nagaoka M, Ogiwara K, et al. Integrin-supported fast rate intracellular delivery of plasmid DNA by extracellular matrix protein embedded calcium phosphate complexes. Biochemistry. 2005; 44:12273-12278.

19. Jeyarajan S, Xavier J, Rao NM, Gopal V. Plasmid DNA delivery into MDA-MB-453 cells mediated by recombinant Her-NLS fusion protein. Int J Nanomedicine. 2010;5:725-733.

20. Thomas JJ, Rekha MR, Sharma CP. Dextran-protamine polycation: An efficient nonviral and haemocompatible gene delivery system. Colloids Surf B Biointerfaces. 2010;81:195-205.

21. Xu ZP, Zeng QH, Lu GQ, Yu AB. Inorganic nanoparticles as carriers for efficient cellular delivery. Chem Eng Sci. 2006;61:1027-1040.

22. Sokolova V, Epple M. Inorganic nanoparticles as carriers of nucleic acids into cells. Angew Chem Int Ed. 2008;47:1382-1395.

23. Zhou C, Yu B, Yang X, et al. Lipid-coated nano-calcium-phosphate (LNCP) for gene delivery. Int J Pharm. 2010;392:201-208.
International Journal of Nanomedicine

\section{Publish your work in this journal}

The International Journal of Nanomedicine is an international, peerreviewed journal focusing on the application of nanotechnology in diagnostics, therapeutics, and drug delivery systems throughou the biomedical field. This journal is indexed on PubMed Central, MedLine, CAS, SciSearch ${ }^{\circledR}$, Current Contents ${ }^{\circledR} /$ Clinical Medicine,

\section{Dovepress}

Journal Citation Reports/Science Edition, EMBase, Scopus and the Elsevier Bibliographic databases. The manuscript management system is completely online and includes a very quick and fair peer-review system, which is all easy to use. Visit http://www.dovepress.com/ testimonials.php to read real quotes from published authors. 